\title{
FOREWORD TO THE ISSUE
}

\author{
SZ. GY. RÉVÉSZ
}

This number 3 volume 44 (September 2018) is again a special issue. As announced in June, here we continue publishing papers dedicated to the memory of Jean-Pierre Kahane (December 11, 1926 - June 21, 2017).

Sergey V. Konyagin and Vitalii V. Arestov were guest editors of our preceding and present issues. 